\title{
Installation cost estimation of IPFC for Power Loss Reduction in Transmission lines using Firefly Algorithm
}

\author{
Vishnu Charan T [1] Alivelu manga Parimi [1] Chandram Karri [2] \\ Department of Electrical and Electronics Engineering \\ [1] BITS Pilani Hyderabad Campus \\ [2] BITS Pilani Goa Campus \\ INDIA
}

\begin{abstract}
Firefly algorithm is proposed in this article for placement of Interline power flow controller (IPFC) to reduce power loss of a transmission line and cost estimation. The IPFC is one of the series FACTS devices. It can control active and reactive power of multiple transmission lines. Power loss reduction, improvement of efficiency of the transmission line and enhancement of voltage profile are major issues in the power transmission system. The average power loss in the system is taken as the objective function with various constraints and then cost is estimated. The code has been developed in MATLAB (Version 2019) and executed on personal computer. The proposed algorithms has been tested on IEEE 5 bus system and the results are presented. It is noticed that the proposedalgorithmprovidesbestresultsintermsofpowerlossandinstallationcostwithlesscomputationaltime.
\end{abstract}

Key-Words: IPFC, firefly algorithm, Installation cost, power loss

Received: March 23, 2020. Revised: September 8, 2020. Accepted: September 14, 2020. Published: October 2, 2020.

\section{Introduction}

Flexible alternating current transmission system (FACTS) devices and controllers are developed to provide an attractive solution for several key issues in transmission and distribution of the large scale power systems [1]. Placement of FACTS controllers in power system network is one of the key research areas in power system planning, operation and control. Thyristor based FACTS devices are earlier employed for the reactive power control in power grid network. Major disadvantage of these devices is formation of resonance in the transmission network[2]. Voltage source inverter (VSI) based FACTS devices nullified the resonance effect in the transmission line. These devices such as STATCOM, SSSC, UPFC and interline power flow controller (IPFC) are versatile and can operates in several modes [3]. Connecting two devices in a transmission line cost of equipment also becomes an additional constraint in practical grids. The IPFC [5] is more suitable for improving the active power and reducing reactive power flow in a multi -transmission line network [11].

\subsection{Literature survey}

Many researchers have suggested various FACTS devices, optimization algorithms [4, 6, 14], control strategies [10] and tuning methods [10] for FACTS devices. For finding the optimal location, various algorithms proposed such as Genetic algorithm [7], Particle swarm optimization [8, 9, 13], Gravitational Search Algorithm [16], Evolutionary programming [17], Evolutionary algorithm [19], a novel method [20], Moth flame algorithm[15] are used in previous studies.

Static VAR compensator(SVC) and TCSC sizing is determined using PSO in identifying the ideal location of IPFC increases voltage stability in the system is discussed in [13]. The genetic algorithm [7] is used for solving the optimization problem. Exact location of the IPFC in a power distribution scheme for improving the voltage stability is also investigated. The GSA [16] determines the magnitude and angle of injecting voltage of the IPFC whereas the genetic algorithm identifies optimal position of IPFC. Newton Raphson method is used in performing the load flow. Output of hybrid algorithm indicates that optimal location of the IPFC leads to improve the system voltage stability. Line utilization factors of congestion management is used for optimal location of the IPFC . Firefly algorithm is used for determining the sizing of the device. The position of IPFC is identified based on the Disparity Line Utilization Factor (DLUF). The DLUF provides difference between per- 
centage of MVA utilization in each line connected to a common bus. The device is located between lines which has the maximum DLUF. After placing the IPFC in a network, firefly algorithm [18] is tuned for varying active power loss, security margins, total voltage variation and capacity of the device.

\subsection{Observations and motivation}

Various heuristic and modern heuristic methods have been proposed for achieving global solutions for different problems in the planning of power system. Reduction of powerloss depends on the selection, placement and tuning of the FACTS device. In brief, the IPFC can be used effectively to control active power and reactive power of multiple transmission lines. Several soft computing methods have already suggested for reduction of the power loss in the transmission line. Quality of the solution depend on various control parameters. It is observed from the literature survey that the FA is a simple and an efficient algorithm. It has been applied for many complex problems in the engineering. The features of the FA motivated to develop the algorithm in this article. The FA is adopted in this article compared to other algorithms because it takes less computuaional for proving the optimal solution.

\subsection{Organization of article}

The proposed FA is implemented in MATLAB (version 2019) and executed on personal computer. The rest of the article is divided in to five sections. Section 2 describes about interline power flow controller. section 3 provides a short description about firefly algorithm. Development of the algorithm is given in section 4. Case studies and Simulation results with discussion is provided in section 5. Conclusions of the work is mentioned in the last section.

\section{Interline power flow controller (IPFC)}

Interline power flow controller (IPFC) [5] is a series FACTS device. Multiple converters provide reactive power compensation in multiple lines connected in the network. The IPFC uses multiple Static Synchronous Series Compensators. schematic diagram of the IPFC device is shown in Fig 01.

Each voltage source converter (VSC) is placed in series with line 1 and line 2. They are interconnected using DC link. This link allows transmission of real power between the transmision lines. Power demand of one line can be compensated by other line. Wih this

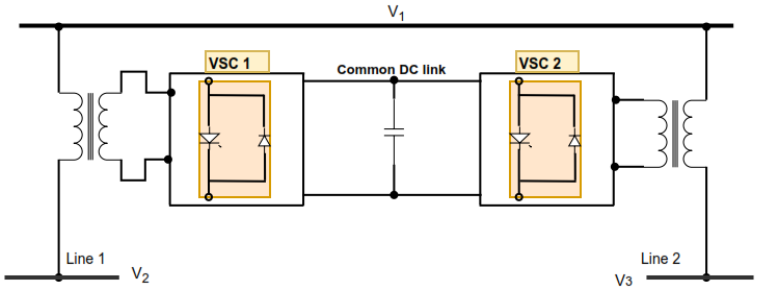

Figure 1: Schematic Diagram of IPFC

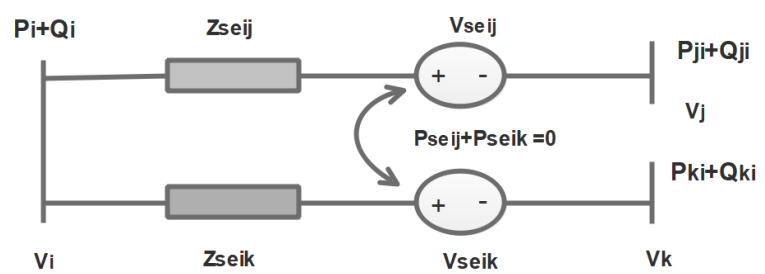

Figure 2: Simplified network of Interline Power Flow Controller in 3 bus system

mechanism, the IPFC control real power and reactive power flow. More information can be found in [20, 21]. Modelling of the IPFC is one of the key issue in load flow for finding the optimal placement. A short description about the modelling of the IPFC for load flow study is given below.

\subsection{Modelling of IPFC for Load Flow Study}

The IPFC consists of two back to back voltage source converters (VSC). It is connected in series with two transmission lines via injected transformer and the DC terminals of the converter. The IPFC can control two transmission line parameters with 3 bus voltages. Equivalent circuit of the IPFC is shown in Fig 02.

In the Fig 02, three buses and two transmission lines are connected with bus $j$ and $k . V_{i}, V_{j}$ and $V_{k}$ are complex bus voltages at lines $i, j$ and $k$ respectively. $V_{1}, \delta_{1}$ are the magnitude and angle of respective bus. $V_{\text {sein }}$ is the complex controllable series injected voltage source which represents the series compensation of the series converter. $V_{\text {sein }}, \delta_{\text {sein }}$ are the magnitude and angle of $V_{\text {sein }}$ respectively. Active power can be exchanged between the two or number of series converters connected with the DC link. Resultant of active power exchange is zero.

\subsection{Incorporation of IPFC in load flow study}

Active power can be exchanged between the two or " $n$ " no. of series converters connected with the DC link. Resultant of active power exchange shall be Zero. Power flow equations are given below. 


\subsection{Bus i}

$$
\begin{gathered}
I_{i}=I_{i j}+I_{i k} \\
I_{i}=\frac{V_{i}-V_{s e i j}-V_{j}}{Z_{s e i j}}+\frac{V_{i}-V_{s e i k}-V_{k}}{Z_{s e i j}}
\end{gathered}
$$

From the above equations, Compleex powers can be derived and the expressions are provided here.

$$
S_{i}=V_{i} I_{i j}^{*}=P_{i}+j Q_{i}
$$

The complex power expression in terms of voltages and impedance is given below.

$$
S_{i}=V_{i}\left[\frac{V_{i}-V_{s e i j}-V_{j}}{Z_{s e i j}}+\frac{V_{i}-V_{s e i k}-V_{k}}{Z_{s e i j}}\right]^{*}
$$

Simplifying the above equation to get the expressions for active and reactive power and these equations can be found in [23].

For solving loadflow with IPFC with operating constraints are considered as follows:

$$
\begin{gathered}
V_{\text {sein }}^{\min } \leq V_{\text {sein }} \leq V_{\text {sein }}^{\max } \\
\pi \leq \theta_{\text {sein }} \leq-\pi
\end{gathered}
$$

The real power exchange between th converter through DC link is provide in the following equation

$$
P_{i}=\sum P_{\text {sum }}=0
$$

IPFC can minimize the real and reactive power flow of line $i-j$ and line $i-k$. Active and reactive power flow of sending end transmission ine which is connected to bus j, k are $P_{i n}$ and $-Q_{n i}$.

The real and reactive power of the IPFC are

$$
\begin{gathered}
P_{n i}-P_{n i}^{s p e c}=0 \\
Q_{n i}-Q_{n i}^{s p e c}=0
\end{gathered}
$$

where $n=j, k P_{n i}^{\text {spec }}, Q_{n i}^{\text {spec }}$ are the specified active and reactive power flow control reference respectively. The method of implementation of IPFC power flow control model in Newton Raphson power flow algorithm. For FACTS branches i-j, $P_{n i}$ and $Q_{n i}$ are controlled. Also, $P_{n i}$ and $Q_{n i}$ balance in respective buses also be maintained. The jocobian matrix is given in [24].

where $\delta P_{i}, \delta Q_{i}, \delta P_{j}, \delta Q_{j}, \delta P_{k}, \delta Q_{k}$ are mismatches at busesi, $j, k$. The first four rows of the jocabean matrix with respect to the IPFC power flow control and active power exchange. The power balnce constraints are

$$
\begin{gathered}
P_{n i}-P_{n i}^{s p e c}=0 \\
Q_{n i}-Q_{n i}^{s p e c}=0 \\
P E=\sum P_{\text {sein }}=0
\end{gathered}
$$

The terms

\subsection{Initialization of control variables of IPFC in Power flow}

In th beginning the algorithm, all bus voltages are assumed as flat voltages. The terms of the last rows of the system Jacobean matrix relate directly to the power mismatch equation at buses $i, j$ and $k$. In the present methodology for the second series converter, it is presumed that the active power flow control constraint is used while the reactive power flow constraint is in idle mode and vice versa. In general, both the scheme can be implemented in the newton power flow equation. From the equations, new IPFC Jacobean matrix will be arrived by applying partial differential method. The relevant equations can be found in $[22,23,24,25,26]$. Voltage expressions are provided in [22].

\subsection{Objective Function}

The objective of this study is to find the optimal location of the IPFC in order to reduce average transmission losses of overall network and minimize respective bus voltage drop in the network. The objective function is

$$
P_{\text {averageloss }}=\sum_{k=1}^{N} \frac{P_{N}}{N}
$$

Here, $P_{N}$ is the power loss of individual transmission line.

$$
V D=\sum_{k=1}^{N}\left|V_{i}-V_{\text {ref }}\right|
$$

These values change for different locations of the IPFC. Since 'N' is the number of lines in the system, the above expression calculates the average value of the real power losses in the lines. 


\subsection{Cost Function of IPFC}

The cost of installation of UPFC is is taken from [7]. The equations are

$$
C_{U P F C}=0.0003 S^{2}-0.2691 S+188.22
$$

$C_{U P F C}=$ Cost of installation of UPFC devices in US dollar perKVAR

$\mathrm{S}=$ Operating range of UPFC device in MVAR

As UPFC is having series and shunt combination of two SSSC devices, the combination of both devices combining together the above cost function is arrived, the above function is derived from the Siemens database. Converting UPFC cost function into IPFC as assuming both are having two SSSC devices like two inverters and two transformers along with the control circuit. Converting above (13) into one combination of inverter, transformer and controller the cost equation of one set is designated as CIPFCA. The IPFC will have two SSSC connected in series circuit as per explained in section 2, the equation of IPFC will be achieved with dividing by 2 of (13). $S_{i}, S_{j}$ are cost functions for converters connected between line $\mathrm{i}$ and $\mathrm{j}$ respectively. $Q_{i 1}, Q_{i 2}$ are reactive power flows in line $\mathrm{i}$ before and after placing of IPFC. $Q_{j 1}, Q_{j 2}$ reactive power flows in line $\mathrm{j}$ before and after placing of IPFC. Using the above cost function, it is understood that IPFC equipment cost directly related to reactive power compensation. Depending on the required compensation of reactive power IPFC equipment cost will be calculated.

$$
\begin{aligned}
& C_{I P F C-A}=0.00015 S_{i}^{2}-0.1345 S_{i}+94.11 \\
& C_{I P F C-B}=0.00015 S_{j}^{2}-0.1345 S_{j}+94.11
\end{aligned}
$$

Using the above cost function, it is understood that the IPFC equipment cost directly related to reactive power compensation. Depending on the required compensation of reactive power the cost of IPFC will be calculated. In this article, Firefly algorithm has been adopted for finding the optimal placement of the interline power flow controller. The detailed information is provided in section 3 .

\section{Firefly algorithm}

Nature is a motivation for understanding several biologically inspired algorithms. survival of fittest is one of the motivations for genetic algorithm. colonial behavior, herding, flocking, etc are some examples. communication is also another motivation. various species communicate with one to another through several ways. Flashing property is utilised by fireflies to communicate. They produce a short flash with a particular order. The light is produced by the bio luminescence. The flashing is applied to attract their partner. A suitable partner will communicate back. It is observed that the light intensity decreases with respect to distance. a firefly receives a response from fireflies around it within a visual range of the flash. Based on these observations, Firefly Algorithm is developed by Xin She Yang in 2008 [27, 28, 29]. The stages involved in the algorithm are provided in the following sub-sections.

\subsection{Development of Firefly algorithm}

When firefly is considered as a solution in the selected solution space, the attraction and movement of fireflies motivate to find the optimal solution. In the process, the candidate fireflies follow the brighter fireflies. Therefore, brightness is considered as the objective function. Initially, all are randomly selected in search space. The FA can be summarized in two stages. The explanation is provided here.

\subsubsection{Variation of light intensity}

Light intensity is the objective function. A firefly with low intensity will attract another firefly with low intensity for minimization.

\subsubsection{Attractiveness}

In the firefly algorithm, the form of attractiveness function of a firefly is the following monotonically decreasing function

$$
\beta(r)=\beta_{0} e^{-\gamma r_{i j}^{2}}
$$

where, $\mathrm{r}$ is the distance between any two fireflies, $\beta_{0}$ is the initial attractiveness at $\mathrm{r}$ and $\gamma$ is an absorption coefficient which controls the decrease of the light intensity.

\subsubsection{Distance}

The distance between any two fireflies $i$ and $j$, at positions $x_{i}$ and $x_{j}$, respectively, can be defined as a Cartesian or Euclidean distance as follows

$$
r_{i, j}^{2}=\left[y_{j}-y_{i}\right]^{2}+\left[x_{j}-x_{i}\right]^{2}
$$

However, the calculation of distance $r$ can also be defined using other distance metrics, based on the nature of the problem, such as Manhattan distance or Mahalanobis distance. 


\subsubsection{Movement}

The movement of a firefly is

$$
x_{i}^{t+1}=x_{i}^{t}+\beta(r)\left(x_{i}^{t}-x_{j}^{t}\right)+\alpha \varepsilon_{i}^{t}
$$

where $\beta(r)$ is the attractiveness function of the firefly and is given by

where the first term is the current position of a firefly, the second term is used for considering a firefly's attractiveness to light intensity seen by adjacent fireflies, and the third term is used for the random movement of a firefly in case there are not any brighter ones.

\section{Development of Firefly algorithm for Optimal placement of IPFC}

The step-by-step procedure for the proposed FA is provided below.

\subsection{Step 01-Input parameters}

(i)Line data and bus data, the bus voltage limits for the given test system. (ii) IPFC parameters. (iii) The control parameters for Firefly algorithm. (iv) Number of generations.

\subsection{Step 02- Initialization}

(i) Randomly initializing the population. Here, the bus voltages are taken as control variables. (ii) IPFC parameters are initialised.

$$
\text { Population }=\left[X_{1}, X_{2}, X_{3}, \ldots . . X\right]
$$

\subsection{Step 03- Evaluation of fitness function}

(i) Ybus is calculated (ii) Injected powers are calculated. (iii) Jacobian matrix is evaluated. (iv) The fitness function is evaluated by determining the average of the active power loss in the connected transmission lines of the system using equation (1). If the tolarence is within the specified limit, then algorithm is terminated. Otherwise, control variables are updated using step 04 .

\subsection{Step 04- Updation of control variables using FA}

(i) Sorting the locations based on the fitness function. Here, fitness function is evaluated by placing the IPFC for all possible locations of the IPFC and then best location of the IPFC is found based on the power loss.
Table 1: Control parameters of the firefly algorithm

\begin{tabular}{ccc}
\hline S No & Control variable & Numerical values \\
\hline 1 & Number fireflies & 25 \\
2 & Generations & 50 \\
3 & $\alpha$ & 0.5 \\
4 & $\beta$ & 0.5 \\
5 & $\gamma$ & 1 \\
\hline
\end{tabular}

Table 2: Lina Data of 5 BUS system

\begin{tabular}{ccccccc}
\hline S No & From & To & r & x & G & B \\
\hline 1 & 1 & 2 & 0.02 & 0.06 & 0 & 0.06 \\
2 & 1 & 3 & 0.08 & 0.24 & 0 & 0.05 \\
3 & 2 & 3 & 0.06 & 0.18 & 0 & 0.04 \\
4 & 2 & 4 & 0.06 & 0.18 & 0 & 0.04 \\
5 & 2 & 5 & 0.04 & 0.12 & 0 & 0.03 \\
6 & 3 & 4 & 0.01 & 0.03 & 0 & 0.02 \\
7 & 4 & 5 & 0.08 & 0.24 & 0 & 0.05 \\
\hline
\end{tabular}

The bus needs to have at least 2 transmission lines. (ii) connected it for a possible location for the IPFC. (iii) Randomly picking new locations in the neighborhoods of the best sites. (iv) Control variables are updated using equation (6), (7) and (8)

\subsection{Step 05- Termination creteria}

The above step 3 and step 4 are repeated until the maximum number of iterations are reached.

\subsection{Step 06- Final results}

Power loss, bus voltages, active and reactive power flows are calculated. Estimation of the cost of IPFC is determined using equation (4) and (5).

\section{Case studies and Simulation re- sults with discussion}

Firefly code is developed in MATLAB (Version 2019). The algorithm has been tested on IEEE 5 BUS system. The objective function is average power loss during execution of the FA. For all cases, the control parameters of the FA are shown in Table 1.

IEEE 5 Bus system is considered to test the effectiveness of the suggested algorithm. The bus data and line data is provided in Table 2 and 3.

The objective function is the average of the power loss in the seven transmission lines during the execution of the proposed algorithm. Initially, NR 
Table 3: Bus Data of 5 BUS system

\begin{tabular}{ccccccc}
\hline S No & BUS & Voltage & P & Q & $P_{G}$ & $Q_{G}$ \\
\hline 1 & Slack & 1.06 & 0.0 & 0 & 0 & 0 \\
2 & PQ & 1.00 & 0.2 & 0.1 & 0 & 0 \\
3 & PQ & 1.00 & 0.45 & 0.15 & 0 & 0 \\
4 & PQ & 1.00 & 0.40 & 0.05 & 0 & 0 \\
5 & PQ & 1.00 & 0.60 & 0.10 & 0 & 0 \\
\hline
\end{tabular}

Table 4: Rotor angles of IEEE 5 BUS system

\begin{tabular}{ccc}
\hline Bus No & Angle without IPFC & Angle with IPFC \\
\hline 1 & 0.0000 & 0.0000 \\
2 & -1.8735 & -1.8037 \\
3 & -4.4466 & -5.668 \\
4 & -4.7531 & -6.3461 \\
5 & -5.43 & -6.0624 \\
\hline
\end{tabular}

method is applied and voltage profiles and transmission line losses, reactive power are calculated without the IPFC. Simulation is run using the Firefly Algorithm for optimal location and the most suitable location is 2 nd bus. Voltages and angles of IEEE 5 BUS system are given Table 4. During the execution of the FA, average power loss is noted and shown in Fig 03. It is note from the table that the algorithm provides the solution within 230 iterations. The minimum power loss is 0.77 . Values of the transmission losses without and with IPFC at BUS 2 are given in Table 05. The average power losses of transmission lines without IPFC is 0.87 p.u and with IPFC is 0.78 p.u.

Reactive power with and without IPFC of IEEE 5 BUS system is given Table 05 .

Voltages at each bus with and without IPFC are shown in Fig 04.

Reactive power at each bus location with and without IPFC are shown in Fig 5.

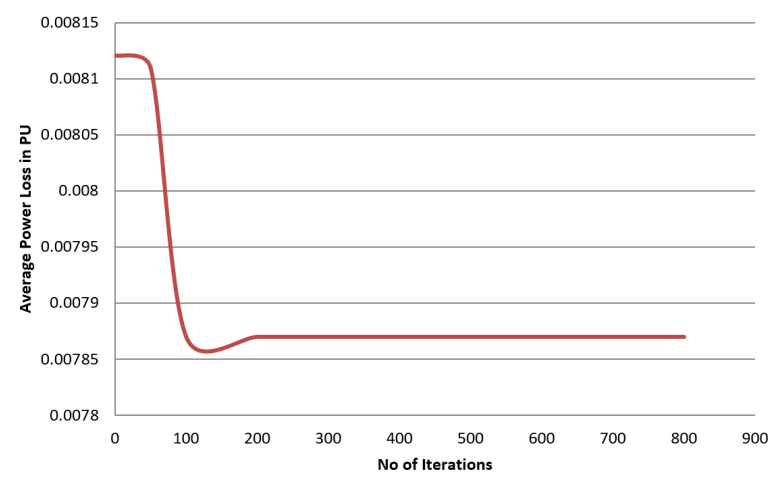

Figure 3: Average Power loss at each iteration by the proposed algorithm for IEEE 5 BUS system
Table 5: Reactive power with and without IPFC of IEEE 5 bus system

\begin{tabular}{ccc}
\hline Line & $Q_{\text {withoutIPFC }}$ & $Q_{\text {withIPFC }}$ \\
\hline $1-2$ & 0.74 & 0.7555 \\
$1-3$ & 0.1682 & 0.1686 \\
$2-3$ & -0.0252 & -0.0229 \\
$2-4$ & -0.0172 & -0.0219 \\
$2-5$ & 0.0556 & 0.0374 \\
$3-4$ & 0.0236 & 0.0223 \\
$4-5$ & 0.0052 & 0.0052 \\
\hline
\end{tabular}

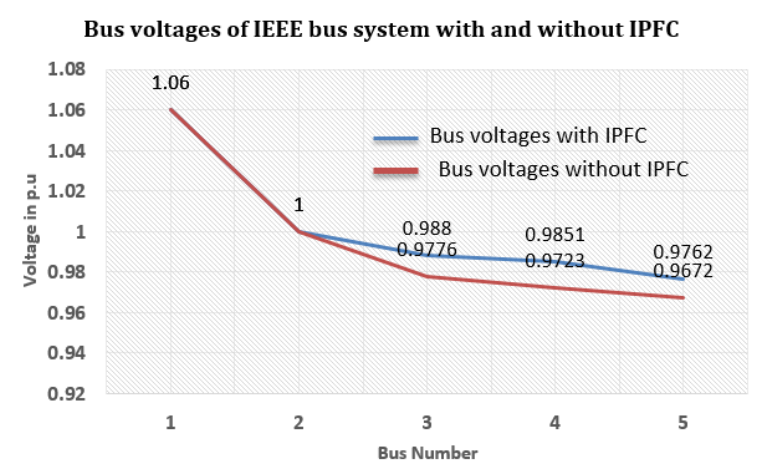

Figure 4: Voltages at each bus with and without IPFC

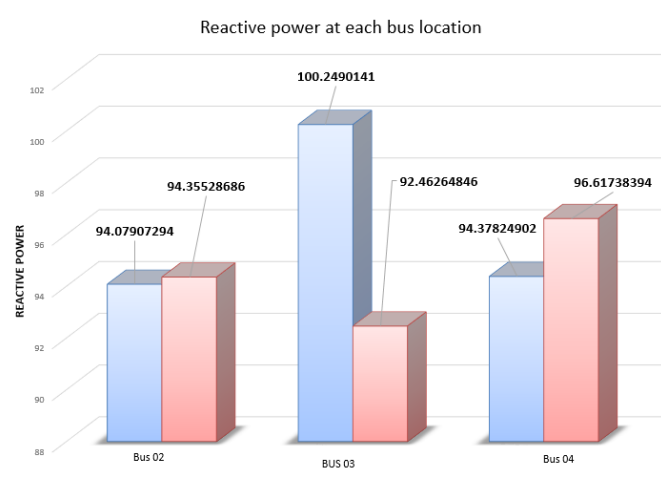

Figure 5: Reactive power at each bus location with and without IPFC for IEEE 5 bus system 
Table 6: Location, average power loss with and without IPFC and cost of IPFC

\begin{tabular}{cccc}
\hline Loc & Loss $_{\text {withoutIPFC }}$ & Loss withIPFC $_{\text {w }}$ & Cost(Rs) \\
\hline Bus 2 & 0.0087 & 0.0078 & 12998.72 \\
\hline
\end{tabular}

\subsubsection{IPFC cost calculation at Bus 2}

At optimal location 2 erection cost of IPFC is calculated with reactive power obtained before and after placement of IPFC at bus 2. The reactive power before and after placement of IPFC is tabulated. As per optimal location of IPFC at bus 2 the two converters are installed in lines between 2 to 3 and 2 to 5 of transmission system. At bus 2 reactive power flow for Q2 to 3 and Q 2 to 5 . The number of iterations for firefly is set to 20. As per the analysis, the FA execution time is $58 \mathrm{sec}$. Location, average power loss with and without IPFC and cost of IPFC calculated using eqn (16),(17) is listed in Table 06.

\section{Conclusions}

The Firefly Algorithm is implemented using MATLAB and tested on IEEE 5 BUS bus system. The results showed that the overall losses in the transmission line of the power system network are reduced by using IPFC and bus voltages are improved. The exact location of the IPFC in the algorithm is determined with the fitness function of average power loss. Once IPFC is placed optimally, the power flows in the transmission lines are also considerably improved. It is observed that the FA converged in a shorter time and it is known as most optimum time to converge for any bus configuration. In addition to the above, cost of optimal location of IPFC is also estimated. Ccost of the IPFC device is derived based on KVAR basis. Depending on requirement of KVAR compensation, cost of IPFC can be achieved. Analysis in this paper provided will be more useful to different grid owners, industries and construction companies for making way forward to install the IPFC devices in a provided grid network.

\section{References:}

[1] Narain G. Hingorani, Laszlo Gyugyi Understanding FACTS: Concepts and Technology of Flexible AC Transmission Systems, Wiley-IEEE Press, December 1999. ISBN 978-0-7803-3455-7

[2] E. Acha, C.R. Fuerte-Esquivel, H. Amirez-Perez, C. Angeles-Camacho, FACTS Modelling and
Simulation in Power Networks, John Wiley and Sons, Chichester, 2004. ISBN: 0-470-85271-2

[3] Xiao-Ping Zhang, Christian Rehtanz, Bikash Pal, Flexible AC Transmission Systems: Modelling and Control, Springer, March 2006. ISBN 9783-540-30606-1

[4] Yang, X. S. (2008). Nature-Inspired Metaheuristic Algorithms. Luniver Press. ISBN 978-1905986-10-1

[5] Gyugyi L, Sen KK, Schauder CD. The interline power flow controller: a new approach to power flow management in transmission systems. IEEE Trans Power Del 1999;14(3) page :1115-23.

[6] Singh, S.N., David, A.K.: 'Optimal location of FACTS devices for congestion management', Electr. Power Syst. Res., 2001, 58, pp. 71-79

[7] L.J. Cai ; I. Erlich ; G. Stamtsis "Optimal choice and allocation of FACTS devices in deregulated electricity market using genetic algorithms" IEEE PES Power Systems Conference and Exposition, 2004, 10-13 Oct. 2004 DOI: 10.1109/PSCE.2004.1397562

[8] Saravanan M, Slochanal SMR, Venkatesh P, Abraham JPS. Application of particle swarm optimization technique for optimal location of FACTS devices considering cost of installation and system loadability. Electr Power Syst Res 2007; 77 page:276-83.

[9] Mohamed, K.H., Rama Rao, K.S., Md. Hasan, K.N.: 'Application of particle swarm optimization and its variants to interline power flow controllers and optimal power flow'. Int. Conf. on Intelligent and Advanced Systems (ICIAS 2010), Kuala Lumpur, Malaysia, 2010, pp. 1-6

[10] Bindeshwar Singh, N.K. Sharma, A.N. Tiwari, "A Comprehensive Survey of Optimal Placement and Coordinated Control Techniques of FACTS Controllers in Multi-Machine Power System Environments", Journal of Electrical Engineering and Technology, vol. 5, pp. 79, 2010.

[11] Frank Kreikebaum, Deepak Divan, Electrical Transmission Systems and Smart Grids, pp. 159, 2013.

[12] Canbing LI, Liwu XIAO, Yijia CAO, Qianlong ZHU, Baling FANG, Yi TAN, Long ZENG, "Optimal allocation of multi-type FACTS devices in power systems based on power flow entropy", Journal of Modern Power Systems and Clean Energy, vol. 2, pp. 173, 2014. 
[13] Siti Amely Jumaat, Ismail Musirin, "Comparison of SVC and TCSC Installation in Transmission Line with Loss Minimization and Cost of Installation via Particle Swarm Optimization", Applied Mechanics and Materials, vol. 785, pp. 495, 2015.

[14] Naganathan Archana, Ranganathan Vidhyapriya, "Improving Voltage Stability of Power System by Optimal Location of FACTS Devices Using Bio-Inspired Algorithms", Circuits and Systems, vol. 07, pp. 805, 2016.

[15] Indrajit N. Trivedi, Pradeep Jangir, Siddharth A. Parmar, Narottam Jangir, "Optimal power flow with voltage stability improvement and loss reduction in power system using Moth-Flame Optimizer", Neural Computing and Applications, 2016.

[16] Biplab Bhattacharyya, Sanjay Kumar, "Loadability enhancement with FACTS devices using gravitational search algorithm", International Journal of Electrical Power and Energy Systems, vol. 78, pp. 470, 2016.

[17] Krishnan B, Ramalingam M, Vellayutham D (2016) Evolutionary programming-based simulation of bilateral real power contracts by optimal placement of flexible AC transmission system devices using contingency analysis. Electr Power Compon Syst 44(7):806-819

[18] Almasi, Omid N. Rouhani, Modjtaba (2016). "A new fuzzy membership assignment and model selection approach based on dynamic class centers for fuzzy SVM family using the firefly algorithm”. Turkish Journal of Electrical Engineering and Computer Sciences. 4: 1-19

[19] SEKHAR A. HEMA, DEVI A. LAKSHMI, "Optimal Sizing and Placement of Advanced SVC in Transmission System by Using Evolutionary Algorithms", i-manager's Journal on Electrical Engineering, vol. 10, pp. 30, 2017.

[20] Van Dai Le, Xinran Li, Peiqiang Li, Cao Quyen Le, "A novel approach for determining optimal number and placement of static var compensator device to enhance the dynamic performance in power systems", Electrical Engineering, 2017.

[21] Ashfaq Hussain, Muhammad Amin, R. D. Khan, Fayyaz Ahmad Chaudhry, "Optimal Allocation of Flexible AC Transmission System Controllers in Electric Power Networks", INAE Letters, vol. 3, pp. 41, 2018.
[22] Serhat Duman, "Solution of the Optimal Power Flow Problem Considering FACTS Devices by Using Lightning Search Algorithm", Iranian Journal of Science and Technology, Transactions of Electrical Engineering, 2019.

[23] X.P. Zhang, Modelling of the interline power flow controller and the generalised unified power flow controller in Newton power flow, IEE Proceedings, Generation, Transmission and Distribution 150 (May (3)) (2003) 268-274.

[24] Zhang, X.P.: 'Modeling of the interline power flow controller and the generalized unified power flow controller in Newton power flow', IEE Proc. Gener. Transm.Distrib. , 2003, 150, (3), pp. 268-274

[25] Kamel S, Jurado F. Power flow analysis with easy modelling of interline power flow controller. Electric Power Syst Res 2014;108:234-44.

[26] Y. Zhang, Y. Zhang, C. Chen,Anovel power injection model of IPFC for power flow analysis inclusive of practical constraints, IEEE Transactions on Power Systems 21 (November (4)) (2006) 1550-1556.

[27] Yang, X.S, Nature-inspired metaheuristic algorithms (Luniver Press, Frome,2008)

[28] Yang, X.S.: 'Firefly algorithm, Levy flights and global optimization', Res. Dev. Intell. Syst. XXVI, 2009, pp. 209-218

[29] Fister I, Yang X-S, Brest J. A comprehensive review of firefly algorithms. Swarm Evol Comput 2013;13:34-46.

\section{Creative Commons Attribution License 4.0 (Attribution 4.0 International, CC BY 4.0)}

This article is published under the terms of the Creative Commons Attribution License 4.0

https://creativecommons.org/licenses/by/4.0/deed.en_US 